\title{
Acoustic Radiation from High-Speed Turbulent Boundary Layers in a Tunnel-like Environment
}

\author{
Lian Duan* \\ Missouri University of Science and Technology, Rolla, MO 65409 \\ Meelan M. Choudhari ${ }^{\dagger}$ \\ NASA Langley Research Center, Hampton, VA 23681 \\ Chao Zhang* \\ Missouri University of Science and Technology, Rolla, MO 65409
}

\begin{abstract}
Direct numerical simulation of acoustic radiation from a turbulent boundary layer in a cylindrical domain will be conducted under the flow conditions corresponding to those at the nozzle exit of the Boeing/AFOSR Mach-6 Quiet Tunnel (BAM6QT) operated under noisy-flow conditions with a total pressure $p_{t}$ of $225 \mathrm{kPa}$ and a total temperature of $T_{t}=430 \mathrm{~K}$. Simulations of acoustic radiation from a turbulent boundary layer over a flat surface are used as a reference configuration to illustrate the effects of the cylindrical enclosure. A detailed analysis of acoustic freestream disturbances in the cylindrical domain will be reported in the final paper along with a discussion pertaining to the significance of the flat-plate acoustic simulations and guidelines concerning the modeling of the effects of an axisymmetric tunnel wall on the noise field.
\end{abstract}

\section{Nomenclature}

$C_{p} \quad$ heat capacity at constant pressure, $\mathrm{J} /(\mathrm{K} \cdot \mathrm{kg})$

$C_{v} \quad$ heat capacity at constant volume, $\mathrm{J} /(\mathrm{K} \cdot \mathrm{kg})$

$H$ shape factor, $H=\delta^{*} / \theta$, dimensionless

$M \quad$ Mach number, dimensionless

$M_{r} \quad$ relative Mach number, $M_{r}=\left(U_{\infty}-U_{b}\right) / a_{\infty}$, dimensionless

$\operatorname{Pr} \quad$ Prandtl number, $\operatorname{Pr}=0.71$, dimensionless

$R \quad$ ideal gas constant, $R=287, \mathrm{~J} /(\mathrm{K} \cdot \mathrm{kg})$

$R e_{\theta}$ Reynolds number based on momentum thickness and freestream viscosity, $\operatorname{Re}_{\theta} \equiv \frac{\rho_{\infty} U_{\infty} \theta}{\mu_{\infty}}$, dimensionless

$R e_{\delta_{2}}$ Reynolds number based on momentum thickness and wall viscosity, $\operatorname{Re}_{\delta_{2}} \equiv \frac{\rho_{\infty} U_{\infty} \theta}{\mu_{w}}$, dimensionless

$R e_{\tau}$ Reynolds number based on shear velocity and wall viscosity, $R e_{\tau} \equiv \frac{\rho_{w} u_{\tau} \delta}{\mu_{w}}$, dimensionless

$R M S$ root mean square

$T$ temperature, $\mathrm{K}$

$T_{r} \quad$ recovery temperature, $T_{r}=T_{\infty}\left(1+0.9 \frac{\gamma-1}{2} M_{\infty}^{2}\right), \mathrm{K}$

$U_{b} \quad$ bulk convection speed, $\mathrm{m} / \mathrm{s}$

$U$ mean streamwise velocity in boundary layer, $\mathrm{m} / \mathrm{s}$

$U_{\infty}$ freestream velocity, $\mathrm{m} / \mathrm{s}$

$p$ pressure, $\mathrm{Pa}$

$u \quad$ streamwise velocity, $\mathrm{m} / \mathrm{s}$

$u_{\tau} \quad$ friction velocity, $\mathrm{m} / \mathrm{s}$

\footnotetext{
${ }^{*}$ Assistant Professor. Member, AIAA

${ }^{\dagger}$ Aerospace Technologist, Computational AeroSciences Branch, M.S. 128. Associate Fellow, AIAA

${ }^{\ddagger}$ Graduate Student. Student Member, AIAA

Copyright (C) 2015 by the American Institute of Aeronautics and Astronautics, Inc. The U.S. Government has a royalty-free license to exercise all rights under the copyright claimed herein for Governmental purposes. All other rights are reserved by the copyright owner.
} 


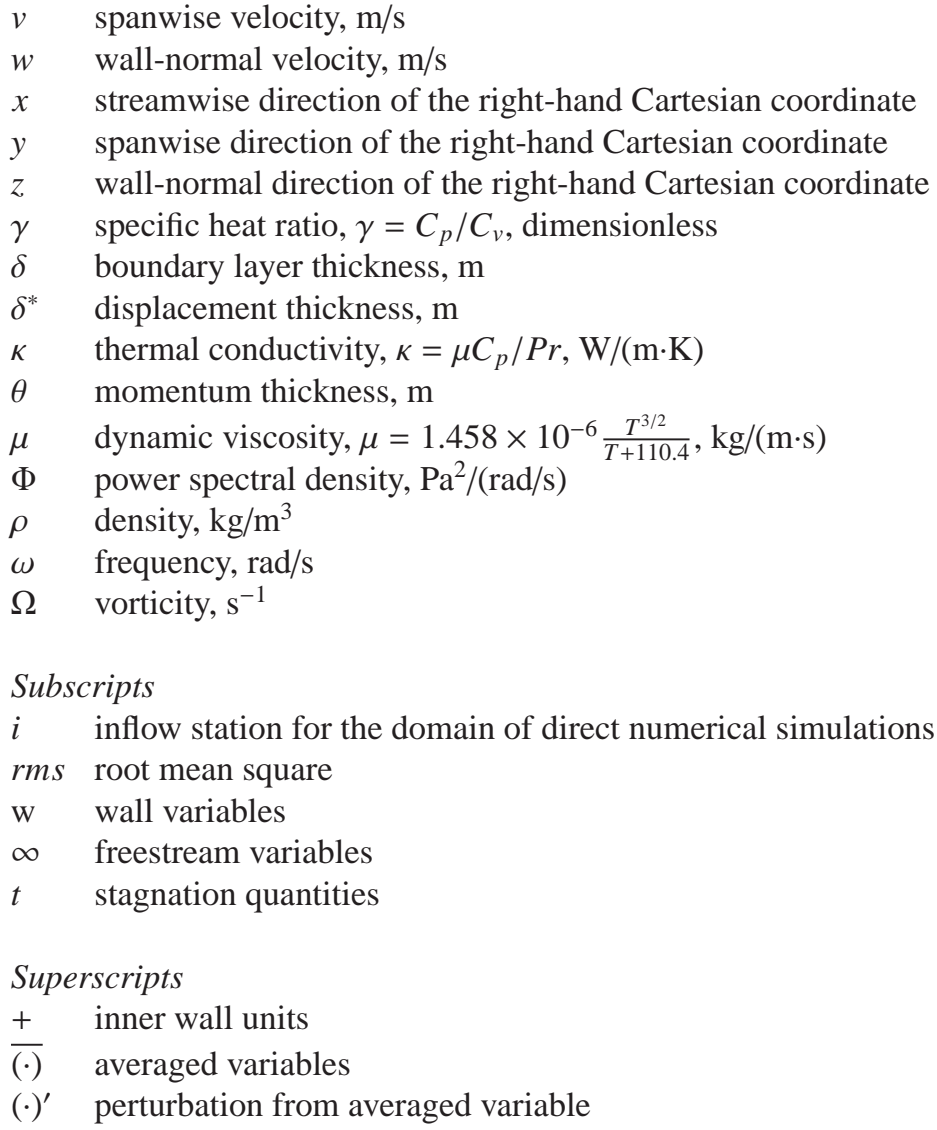

\section{Introduction}

Prediction of laminar-turbulent transition is a critical part of the design of hypersonic vehicles because of the large increase in skin-friction drag and surface heating associated with the onset of transition. Despite continued advances in transition research, the physics of boundary layer transition over these vehicles is not fully understood due to the lack of detailed experimental measurements. Transition testing in conventional (i.e., noisy) wind tunnels has been an important avenue to understanding the transition behavior of hypersonic vehicles, despite the common knowledge that conventional wind-tunnel facilities cannot reliably simulate the in-flight transition behavior over a smooth surface due to the effects of the elevated levels of freestream disturbances. ${ }^{1-3}$ Recent evidence suggests that freestream disturbances may also influence the accelerated onset of transition caused by isolated roughness elements on a nominally smooth surface. ${ }^{4}$ Transition measurements in low disturbance (i.e., quiet) wind tunnels better mimic the in-flight transition characteristics. However, because of the size and Reynolds number limitations of the existing quiet facilities, conventional tunnels will continue to be employed for the testing and evaluation of hypersonic vehicles, especially during ground tests involving large-scale models.

Facility disturbances in conventional tunnels can impact not only the transition location but, possibly, the transition mechanism as well. As a result, the existing methodology to extrapolate wind-tunnel transition results to flight is rather crude and requires substantial improvement. To enable more effective use of the transition data from conventional facilities and permit more accurate extrapolation of the wind-tunnel results to flight, an in-depth knowledge of the broadband disturbance environment in those facilities must be developed.

In unheated tunnels with adequate flow conditioning, the acoustic disturbances are likely to dominate the overall disturbance environment at Mach numbers of 2.5 or above, ${ }^{5-7}$ and their effect on transition cannot be quantified in terms of a single metric corresponding to the root-mean-square amplitude of the freestream disturbances as indicated by the measurements at Purdue University. ${ }^{3}$ With the exception of the early measurements of freestream pressure fluctuations by Laufer ${ }^{5}$ and a few others, ${ }^{2,8}$ there are few measurements that are detailed enough to be suitable for either comparing with computational predictions or for developing models that can be used towards more reliable transition models. The measurements are typically susceptible to experimental errors due to the poor spatial resolution and/or limited frequency response of pressure transducers. ${ }^{9}$ Theoretical models for acoustic radiation from a supersonic 
boundary layer were developed by Phillips ${ }^{10}$ and Ffowcs-Williams and Maidanik, ${ }^{11}$ which attributed a major cause of the acoustic radiation to eddy Mach waves from boundary-layer turbulence convecting supersonically with respect to the free stream. However, a lack of adequate knowledge concerning the boundary-layer turbulence restricted the theoretical predictions to the intensity of the freestream acoustic fluctuations alone.

Direct numerical simulation (DNS) is a valuable tool that can overcome some of the aforementioned difficulties with both experimental measurements and theory and, hence, provide access to both flow and acoustic quantities that are difficult to obtain otherwise. In our previous efforts, DNS have been conducted for flat-plate boundary layers under Mach 2.5 and Mach 6, with nearly adiabatic and cold walls. ${ }^{12-15}$ These flat-plate simulations have the benefits of more easily isolating the acoustic radiation from a single surface, thus facilitating a comprehensive understanding of the freestream disturbance field and its dependence on boundary-layer parameters (e.g., freestream Mach number, wall temperature, Reynolds number). Most hypersonic wind tunnels are, however, axisymmetric. As a result, the freestream disturbances in the tunnel environment reflect the combined outcome of acoustic radiation from all regions of the tunnel wall. The motivation behind the present study is to help enable practical applications of the simulation data for freestream disturbances in the context of actual wind-tunnel experiments and to guide the measurement of tunnel disturbances in high-speed facilities. To simulate the acoustic radiation within an enclosed environment while simultaneously facilitating a direct comparison with acoustic radiation from a single flat wall in an unconfined setting, this paper focuses on investigating the effects of a cylindrical test section on the interior noise field. An attempt will also be made to understand where the previous flat-plate acoustic simulations are applicable, and where, if at all, the effects of an axisymmetric enclosure on the noise field need to be taken into account.

The paper is structured as follows. The flow conditions and numerical methods are outlined in Section II. Section III presents reference results for the characteristics of freestream pressure field induced by flat-plate turbulent boundary layers. A summary of the overall findings is given in Section IV.

\section{Flow Conditions and Numerical Methodology}

\section{A. Flow Conditions and Simulation Domain}

Figure 1 shows a sketch of the computational domain with relevant flow conditions summarized in Table 1 . The flow conditions are similar to those at the nozzle exit of the Boeing/AFOSR Mach-6 Quiet Tunnel (BAM6QT) operated under noisy-flow conditions with a total pressure $p_{t}$ of $225 \mathrm{kPa}$ and an initial total temperature of $T_{t}=430 \mathrm{~K} .3,16$

Under the selected flow condition, measurement of boundary-layer profiles has been conducted on the nozzle wall of BAM6QT by Skotch ${ }^{16}$ and will be available for comparison with the current DNS results. To simulate the acoustic radiation within an enclosed environment while simultaneously facilitating a direct comparison with acoustic radiation from a single flat wall in an unconfined setting, we consider a cylindrical domain with the radius the same as the nozzle exit of BAM6QT. The cylindrical domain is used to allow for the study of the effect of tunnel-like geometry on noise generation and propagation. In addition, by choosing a cylindrical geometry, the effects of surface curvature and pressure gradient in the streamwise direction can be neglected, thus avoiding extraneous complexity in the simulation. Under the chosen flow condition, the ratio of the tunnel radius to the tunnel-wall boundary-layer thickness is $R / \delta \approx 3$. The modest value of $R / \delta$ also makes it easier to propagate high-frequency acoustic waves over large axial distances.

\section{B. Numerical Methods}

The full three-dimensional compressible Navier-Stokes equations in conservation form are solved in cylindrical coordinates. To handle the singularities arising at the polar axis of the governing flow equations, a technique based on power series expansions will be used, ${ }^{17}$ which has been shown to enable the computation of nonaxisymmetric flows in cylindrical coordinates by using highly accurate finite-difference schemes on nonstaggered grids.

The working fluid is assumed to be a perfect gas and the usual constitutive relations for a Newtonian fluid are used. The viscous stress tensor is linearly related to the rate-of-strain tensor, and the heat flux vector is linearly related to the temperature gradient through the Fourier's law. A 7th-order weighted essentially non-oscillatory (WENO) scheme ${ }^{18,19}$ is used to compute the convective flux terms. For the viscous flux terms, a 4th-order central difference scheme is used. The 3rd-order low storage Runge-Kutta scheme by Williamson ${ }^{20}$ is used for time integration.

The turbulent inflow is generated using the recycling/rescaling method developed by Xu and Martin. ${ }^{21}$ Dynamical translation operations ${ }^{22}$ are applied to the recycled turbulence plane at randomly-distributed time intervals to improve the low-frequency characteristics of recycling/rescaling inflow turbulence generation. On the wall, no-slip conditions are applied for the three velocity components and an isothermal condition is used for the temperature with $T_{w} \approx 0.76 T_{r}$. At the outlet boundaries, unsteady non-reflecting boundary conditions based on Thompson ${ }^{23}$ are imposed. Periodic 
boundary conditions are used in the spanwise direction. Domain mapping is used ${ }^{17}$ so that the points on the polar axis become regular interior points and the radial derivative at the origin is calculated with the same order of accuracy as that in the rest of the domain.

\section{DNS of High-Speed Turbulent Boundary Layers over a Flat Plate}

Simulations of acoustic radiation from a turbulent boundary layer over a flat plate are used to provide the basis for comparison with acoustic radiation in a tunnel-like environment. An initial simulation for $M_{\infty}=5.85$ was presented in a previous study. ${ }^{13}$ Additional simulations have now been carried out using a refined grid and longer computational domain to validate those findings and, also, to provide additional data that was not available previously.

Relevant flow conditions are summarized in Table 2, which provides the freestream Mach number, density, and temperature $\left(M_{\infty}, \rho_{\infty}\right.$, and $T_{\infty}$, respectively) as well as the boundary layer thickness and various Reynolds numbers at a selected location where the turbulence statistics are gathered. The flow conditions for the Mach 6 simulation are similar to the operating conditions of the Boeing/AFOSR Mach 6 Quiet Tunnel, and Figure 2 shows the general computational set-up for this simulation. To illustrate the effects of Mach number, conditions for a previous DNS at Mach $2.5^{12}$ are also included in Table 2.

The details of the DNS methodology in general curvilinear coordinates have been documented in Duan et al. ${ }^{12-14}$ The DNS solver has been previously shown to be suitable for computing transitional and fully turbulent flows, including hypersonic turbulent boundary layers, ${ }^{24,25}$ the propagation of linear instability waves in $2 \mathrm{D}$ high-speed boundary layers, and the secondary instability and laminar breakdown of swept-wing boundary layers. ${ }^{26,27}$

Table 3 lists the freestream values of several fluctuating flow variables for the Mach 2.5 and Mach 6 DNS, respectively. The normalized velocity fluctuations at Mach 6 are larger than those at Mach 2.5. Yet, the rms fluctuations in either velocity component are less than approximately $0.2 \%$. Therefore, the dynamics of acoustic fluctuations in the free stream is still likely to be linear in nature over the propagation distances of interest in a wind-tunnel facility. However, further work is necessary to establish the linearity of the acoustic field. The fluctuations in thermodynamic variables are stronger than the velocity fluctuations and also increases in amplitude from Mach 2.5 to Mach 6 . At Mach 6, the rms pressure fluctuations are approximately $2 \%$ of the mean pressure value. Moreover, the numerical results show that $s_{r m s}^{\prime} / R<<p_{r m s}^{\prime} / \bar{p}$, and by using the values listed in Table 3, the following isentropic relations $T_{r m s}^{\prime} / \bar{T} \approx((\gamma-1) / \gamma)\left(p_{r m s}^{\prime} / \bar{p}\right)$ and $\rho_{r m s}^{\prime} / \bar{\rho} \approx(1 / \gamma)\left(p_{r m s}^{\prime} / \bar{p}\right)$ are satisfied, indicating that the freestream fluctuations are nearly isentropic and that the contribution from the entropy mode is minimal. The relative importance of the acoustic mode and the vorticity mode in the free stream is demonstrated by the ratio of dilatation variance $\overline{\left(\partial u_{i} / \partial x_{i}\right)^{\prime 2}}$ and vorticity variance $\overline{\Omega_{i}^{\prime} \Omega_{i}^{\prime}}$, which are representative of the acoustic and vorticity mode, respectively. The large values of $\overline{\left(\partial u_{i} / \partial x_{i}\right)^{\prime 2}} / \overline{\Omega_{i}^{\prime} \Omega_{i}^{\prime}}$ from Table 3 imply the dominance of the acoustic mode over the vorticity mode in the free stream.

The dominance of the acoustic mode over the entropy and vorticity modes confirms that a purely acoustic field in the free stream is successfully isolated by the DNS. In typical high-speed (noisy) wind tunnels, however, multiple disturbance sources exist and all three modes contribute to the freestream fluctuations. ${ }^{2,28,29}$ The DNS thus provide an opportunity to study the generic spectral features and production mechanisms particular to the acoustic disturbance.

Figure 3 shows that the normalized pressure fluctuation intensity $p_{r m s}^{\prime} / \tau_{w}$ increases with freestream Mach number, consistent with the trend predicted by the experimental data reported by Laufer. ${ }^{5}$

Figures $4 \mathrm{a}$ and $4 \mathrm{~b}$ show the pressure spectrum at the wall and in the free stream, respectively. The pressure spectrum is normalized so that the area under each curve is equal to unity. For reference, straight lines with slopes of $2,-1,-7 / 3$, and -5 are also included to gauge the rate of spectral roll-off across relatively low, mid, high and very high (i.e., near Kolmogorov scale) frequencies, respectively. The wall-pressure spectrum compares well with the experimental measurements in a low-speed boundary layer by Farabee and Casarella ${ }^{30}$ and in a Mach 2 supersonic boundary layer by Beresh et al., ${ }^{9}$ as well as with the DNS results by Bernardini and Pirozzoli. ${ }^{31}$ The general features of the freestream spectrum compare well with the measurements by Laufer. ${ }^{5}$ Similar to the wall pressure, the freestream pressure fluctuations for both Mach number cases become weakly constant as $\omega \rightarrow 0$ within the range of the plot and exhibit an approximately $\omega^{-5}$ roll-off at high frequencies predicted theoretically by Blake. ${ }^{32}$ For $\omega \delta / U_{\infty}>3$, the freestream spectrum for the Mach 6 case has significantly higher energy than that in the lower Mach number case. The $-7 / 3$ scaling has been shown to apply to the pressure fluctuations generated by turbulence-turbulence interaction within the inertial subrange of velocity fluctuations in a low-speed turbulent flow. ${ }^{33}$ While the freestream spectrum for the Mach 2.5 DNS and the experiments by Masutti et al. ${ }^{29}$ has an observable region of slope close to $-7 / 3$, a similar region is less evident for the Mach 6 DNS. Further information will be provided through the proposed effort to help identify the generic spectral features, if any, of the acoustic radiation from high-speed boundary layers.

Figure 5 shows a preliminary comparison of DNS results with the wind-tunnel measurement and the calculation 
using Harris Boundary-layer code ${ }^{34}$ for a Mach 5.8 turbulent boundary layer on the nozzle wall of BAM6QT under noisy-flow conditions $\left(R e=9.69 \times 10^{6} / \mathrm{m}, P_{t, \infty}=965 \mathrm{kPa}, T_{t, \infty}=429 \mathrm{~K}\right)$. The DNS and experiments agree well with each other in terms of both boundary-layer profile and wall-pressure spectrum. In comparison, the Mach number profile based on the boundary layer code exhibits larger differences from the measurement and the DNS in the outer part of the boundary layer. Moreover, Figure 5c and Figure 5d show that DNS successfully extends the measured spectra to higher frequencies. The resolution of the high-frequency region as well as the acoustic radiation due to these high-frequency fluctuations are especially important for studying the receptivity process associated with secondmode waves in hypersonic wind tunnels. More refined comparisons are currently ongoing.

To illustrate the distribution of energy among various frequencies, Figure 6 shows the scaled pre-multiplied pressure spectra at selected heights above the surface for the Mach 6 case. The pressure spectra in the inner layer (including the wall, buffer layer, and log layer) have a dominant hump centered on $\omega \delta / U_{\infty} \approx 8$ (or $f \delta / U_{\infty} \approx 1$ ), which is the characteristic frequency of the energetic vortical structures within the boundary layer. Away from the wall into the outer layer, the peak gradually shifts to lower frequencies as spatial intermittency becomes more important. In the free stream, where the pressure signal is predominantly acoustic, the peak of the spectrum is centered at a frequency of $\omega \delta / U_{\infty} \approx 3$ (i.e., $f \approx 108 \mathrm{KHz}$ ), indicating that the characteristic frequency of the acoustic mode is significantly lower than that of the vorticity mode. Similar variation in pre-multiplied pressure spectrum with wall-normal distance is observed for the Mach 2.5 case. The dominance of lower frequencies in the freestream spectrum is consistent with the measurements by Laufer ${ }^{5}$ and needs to be accounted for by any mechanisms that correctly describe the boundary layer radiation.

The space-time correlation contours of the freestream pressure fluctuations are shown in Figure 7a. The skewed shape of the contours at both locations indicates the convective nature of the pressure field. The overall larger inclination of the space-time correlation contours for the Mach 6 case indicates that the convection velocity for the Mach 6 case is larger than the Mach 2.5 case, at least at low to intermediate frequencies. The freestream bulk convection velocity computed using the space-time correlation coefficients is $U_{b} \approx 0.4 U_{\infty}$ for Mach 2.5 and $U_{b} \approx 0.63 U_{\infty}$ for Mach 6, which are close to the experimentally measured values reported by Laufer. ${ }^{5}$ Both the current DNS and the experimental measurements by Laufer ${ }^{5}$ show that the bulk convection speed in the free stream increases with freestream Mach number (Figure 7b). All the freestream bulk convection speeds fall within the region where $M_{r}>1$, with $M_{r} \equiv\left(U_{\infty}-U_{b}\right) / a_{\infty}$, which is expected from the 'Mach wave radiation' concept. The convection velocity is related to the directionality of the acoustic radiation and is an important characteristic of the stochastic acoustic field in the free stream from the standpoint of transition analysis, because the receptivity characteristics are known to be sensitive to the orientation of the plane wave disturbance. ${ }^{35}$

Figures $8 \mathrm{a}$ and $8 \mathrm{~b}$ plot the contours of density gradient magnitude, which mimic the schlieren flow visualization during an experiment. The wave fronts of the radiated disturbances are shown to be inclined within a narrow range of angles with respect to the flow direction. Such a preferred directionality of the freestream waves is consistent with the 'Mach wave radiation' concept. The fact that the radiation wave front for the Mach 6 case is shallower than the Mach 2.5 case is also consistent with the experimentally measured trend. ${ }^{5}$

The above results form the basis for comparison with the acoustic field within an enclosed environment. Results for the latter will be included in the final paper.

\section{Summary}

This abstract presented the feasibility of DNS for capturing the freestream acoustic pressure fluctuations and successfully isolating a purely acoustic freestream disturbance field in the canonical setting of a turbulent boundary layer above a single tunnel wall, and showed good agreement with past experimental results. The modified methodology for extending the existing flat-plate acoustic simulations to a tunnel-like (axisymmetric) environment is also introduced.

The full paper will provide a detailed analysis of acoustic freestream disturbances in a cylindrical domain to mimic the radiation field in the actual wind-tunnel experiment, and discuss where the existing flat-plate acoustic simulations are applicable, and where, if at all, the effects of axisymmetry on the noise field need to be taken into account. These findings will pave the way for more practical application of the simulation data in the context of actual wind-tunnel experiments.

\section{Acknowledgments}

This material is based on the work supported by the Air Force Office of Scientific Research Young Investigator Program and National Aeronautics and Space Administration, Langley Research Center under the Research Coopera- 
tive Agreement No. NNL09AA00A (subcontracted through the National Institute of Aerospace). The authors would like to thank Prof. Pino Martín of the University of Maryland for providing the original code that is modified for the current study.

\section{References}

${ }^{1}$ Pate, S. R. and Schuller, C. J., "Radiated Aerodynamic Noise Effects on Boundary Layer Transition in Supersonic and Hypersonic Wind Tunnels," AIAA Journal, Vol. 7, No. 3, 1969, pp. 450-457.

${ }^{2}$ Donaldson, J. and Coulter, S., "A Review of Free-Stream Flow Fluctuation and Steady-State Flow Quality Measurements in the AEDC/VKF Supersonic Tunnel A and Hypersonic Tunnel B," AIAA Paper 95-6137, 1995.

${ }^{3}$ Schneider, S. P., "Effects of High-Speed Tunnel Noise on Laminar-Turbulent Transition," Journal of Spacecraft and Rockets, Vol. 38, No. 3, 2001, pp. 323-333.

${ }^{4}$ Borg, M. P. and Schneider, S. P., "Effect of Free-stream Noise on Roughness-Induced Transition for the X-51A Forebody," Journal of Spacecraft and Rockets, Vol. 45, No. 6, 2008, pp. 1106-1116.

${ }^{5}$ Laufer, J., "Some Statistical Properties of the Pressure Field Radiated by a Turbulent Boundary Layer," Physics of Fluids, Vol. 7, No. 8, 1964, pp. 1191-1197.

${ }^{6}$ Stetson, K. F., "Nosetip Bluntness Effects on Cone Frustrum Boundary-Layer Transition in Hypersonic Flow," AIAA Paper 83-1763, 1983.

${ }^{7}$ Bushnell, D. M., "Notes on Initial Disturbance Fields for the Transition Problem," Instability and Transition, edited by M. Y. Hussaini and R. G. Voigt, Springer-Verlag, Berlin, Vol. 1, 1990, pp. 217-232.

${ }^{8}$ Kendall, J. M., "Supersonic Boundary Layer Transition Studies," Space Program Summary, Vol. 3, 1970, pp. $43-47$.

${ }^{9}$ Beresh, S. J., Henfling, J. F., Spillers, R. W., and Pruett, B. O. M., "Fluctuating Wall Pressures Measured beneath a Supersonic Turbulent Boundary Layer," Physics of Fluids, Vol. 23, No. 7, 2011.

${ }^{10}$ Phillips, O. M., "On the Generation of Sound by Supersonic Turbulent Shear Layers," Journal of Fluid Mechanics, Vol. 9, 1960, pp. 1-28.

${ }^{11}$ Ffowcs-Williams, J. E. and Maidanik, G., "The Mach wave field radiated by supersonic turbulent shear flows," Journal of Fluid Mechanics, Vol. 21, 1965, pp. 641-657.

${ }^{12}$ Duan, L., Choudhari, M. M., and Wu, M., "Numerical Study of Pressure Fluctuations due to High-Speed Turbulent Boundary Layers," AIAA Paper 2012-3070, 2012.

${ }^{13}$ Duan, L. and Choudhari, M. M., "Numerical Study of Pressure Fluctuations due to a Mach 6 Turbulent Boundary Layer," AIAA Paper 2013-0532, 2013.

${ }^{14}$ Duan, L., Choudhari, M. M., and Wu, M., "Numerical Study of Acoustic Radiation due to a Supersonic Turbulent Boundary Layer," Journal of Fluid Mechanics, Vol. 746, 2014, pp. 165-192.

${ }^{15}$ Duan, L. and Choudhari, M. M., "Analysis of Numerical Simulation Database for Pressure Fluctuations Induced by High-Speed Turbulent Boundary Layers," To appear in the Proceeding of 44th AIAA Fluid Dynamics Conference, June 16-20, 2014, Atlanta, Georgia, 2014.

${ }^{16}$ Skoch, C. R., Disturbances from Shock/Boundary-Layer Interactions Affecting Upstream Hypersonic Flow, Ph.D. thesis, Purdue University, West Lafayette, IN, USA, 2005.

${ }^{17}$ Constantinescu, G. S. and Lele, S. K., "A Highly Accurate Technique for the Treatment of Flow Equations at the Polar Axis in Cylindrical Coordinates Using Series Expansions," Journal of Computational Physics, Vol. 183, 2002, pp. 165-186.

${ }^{18}$ Jiang, G. S. and Shu, C. W., "Efficient Implementation of Weighted ENO Schemes," Journal of Computational Physics, Vol. 126, No. 1, 1996, pp. 202-228.

${ }^{19}$ Taylor, E. M., Wu, M., and Martín, M. P., "Optimization of Nonlinear Error Sources for Weighted Non-Oscillatory Methods in Direct Numerical Simulations of Compressible Turbulence," Journal of Computational Physics, Vol. 223, No. 1, 2006, pp. $384-397$.

${ }^{20}$ Williamson, J., "Low-Storage Runge-Kutta Schemes," Journal of Computational Physics, Vol. 35, No. 1, 1980, pp. 48-56.

${ }^{21} \mathrm{Xu}, \mathrm{S}$. and Martín, M. P., "Assessment of Inflow Boundary Conditions for Compressible Turbulent Boundary Layers," Physics of Fluids, Vol. 16, No. 7, 2004, pp. 2623-2639.

${ }^{22}$ Morgan, B., Larsson, J., Kawai, S., and Lele, S. K., "Improving Low-Frequency Characteristics of Recycling/Rescaling Inflow Turbulence Generation," AIAA Journal, Vol. 49, No. 3, 2011, pp. 582-597.

${ }^{23}$ Thompson, K. W., “Time Dependent Boundary Conditions for Hyperbolic Systems," Journal of Computational Physics, Vol. 68, No. 1, Jan. 1987, pp. 1-24.

${ }^{24}$ Martín, M., "DNS of Hypersonic Turbulent Boundary Layers. Part I: Initialization and Comparison with Experiments," Journal of Fluid Mechanics, Vol. 570, 2007, pp. 347-364.

${ }^{25}$ Duan, L., Beekman, I., and Martín, M. P., "Direct Numerical Simulation of Hypersonic Turbulent Boundary Layers. Part 3: Effect of Mach Number," Journal of Fluid Mechanics, Vol. 672, 2011, pp. 245-267.

${ }^{26}$ Duan, L., Choudhari, M. M., Li, F., and Wu, M., "Direct Numerical Simulation of Transition in a Swept-Wing Boundary Layer," AIAA Paper 2013-2617, 2013.

${ }^{27}$ Choudhari, M. M., Li, F., Duan, L., Chang, C.-L., Carpenter, M. H., Streett, C. L., and Malik, M. R., "Towards Bridging the Gaps in Holistic Transition Prediction via Numerical Simulations (Invited)," AIAA Paper 2013-2718, 2013.

${ }^{28}$ Bounitch, A., Lewis, D. R., and Lafety, J. F., "Experimental Study of Second-Mode Instabilities on a 7-Degree Cone at Mach 6," AIAA Paper 2011-1200, 2011.

${ }^{29}$ Masutti, D., Spinosa, E., Chazot, O., and Carbonaro, M., "Disturbance Level Characterization of a Hypersonic Blow-Down Facility," AIAA Journal, Vol. 50, No. 12, 2013, pp. 2720-2730.

${ }^{30}$ Farabee, T. and Casarella, M. J., "Spectral Features of Wall Pressure Fluctuations Beneath Turbulent Boundary Layers," Physics of Fluids, Vol. 3, 1991, pp. 2410-2420

${ }^{31}$ Bernardini, M. and Pirozzoli, S., "Wall Pressure Fluctuations beneath Supersonic Turbulent Boundary Layers," Physics of Fluids, Vol. 23 , No. 085102, 2011

${ }^{32}$ Blake, W. K., Mechanics of Flow-Induced Sound and Vibration, Academic Press, Orlando, Florida, 1986. 
${ }^{33}$ George, W. K., Beuther, P. D., and Arndt, R. E. A., "Pressure Spectra in Turbulent Free Shear Flows," Journal of Fluid Mechanics, Vol. 148, 1984, pp. 155-191.

${ }^{34}$ Harris, J. and Blanchard, D., "Computer Program for Solving Laminar, Transitional, or Turbulent Compressible Boundary-Layer Equations for Two-Dimensional and Axisymmetric Flow," NASA-TM-83207, 1982.

${ }^{35}$ Fedorov, A. V., "Receptivity of a High-Speed Boundary Layer to Acoustic Disturbances," Journal of Fluid Mechanics, Vol. 491, 2003, pp. 101-129.

${ }^{36}$ Steen, L. E., Characterization and Development of Nozzles for a Hypersonic Quiet Wind Tunnel, Master's thesis, Purdue University, West Lafayette, IN, USA, 2010.

${ }^{37}$ Casper, K. M., “Turbulent Pressure Fluctuations in a Hypersonic Boundary Layer,” AAE 626 Final Project Report, Purdue University, West Lafayette, IN, USA, 2011. 
Table 1. Freestream conditions for Mach 6 DNS of turbulent boundary layers.

\begin{tabular}{cccc}
$M_{\infty}$ & $U_{\infty}(\mathrm{m} / \mathrm{s})$ & $\rho_{\infty}\left(\mathrm{kg} / \mathrm{m}^{3}\right)$ & $T_{\infty}(\mathrm{K})$ \\
5.86 & 870.4 & 0.0105 & 55.0 \\
\hline
\end{tabular}

Table 2. Boundary layer properties at the station selected for the analysis of the acoustic field for the current Mach 6 DNS and Mach 2.5 DNS.

\begin{tabular}{lccccccccccc}
$M_{\infty}$ & $U_{\infty}(\mathrm{m} / \mathrm{s})$ & $\rho_{\infty}\left(\mathrm{kg} / \mathrm{m}^{3}\right)$ & $T_{\infty}(\mathrm{K})$ & $T_{w}(\mathrm{~K})$ & $R e_{\theta}$ & $R e_{\tau}$ & $R e_{\delta 2}$ & $\theta(\mathrm{mm})$ & $\mathrm{H}$ & $\delta(\mathrm{mm})$ & $\delta_{i}(\mathrm{~mm})$ \\
2.5 & 823.6 & 0.1 & 270.0 & 568.0 & $2,834.8$ & 509.9 & $1,656.9$ & 0.583 & 4.14 & 7.69 & 4.0 \\
5.85 & 870.2 & 0.039 & 55.0 & 300.1 & $9,659.2$ & 464.2 & $1,783.3$ & 0.968 & 13.6 & 24.4 & 13.8 \\
\hline
\end{tabular}

Table 3. The disturbance field in the free stream for the Mach 2.5 and Mach 6 DNS.

\begin{tabular}{ccccccccc}
\hline$M_{\infty}$ & $u_{r m s}^{\prime} / \bar{u}$ & $v_{r m s}^{\prime} / \bar{u}$ & $w_{r m s}^{\prime} / \bar{u}$ & $p_{r m s}^{\prime} / \bar{p}$ & $\rho_{r m s}^{\prime} / \bar{\rho}$ & $T_{r m s}^{\prime} / \bar{T}$ & $\frac{\overline{\left(\partial u_{i} / \partial x_{i}\right)^{2}}}{\Omega_{i}^{\prime} \Omega_{i}^{\prime}}$ & $s_{r m s}^{\prime} / R$ \\
2.5 & $7.56 \times 10^{-4}$ & $4.90 \times 10^{-4}$ & $8.09 \times 10^{-4}$ & $3.96 \times 10^{-3}$ & $2.83 \times 10^{-3}$ & $1.13 \times 10^{-3}$ & 1797.8 & $2.19 \times 10^{-4}$ \\
5.85 & $12.6 \times 10^{-4}$ & $9.90 \times 10^{-4}$ & $20.0 \times 10^{-4}$ & $20.6 \times 10^{-3}$ & $14.7 \times 10^{-3}$ & $5.86 \times 10^{-3}$ & 1131.2 & $1.75 \times 10^{-4}$ \\
\hline
\end{tabular}




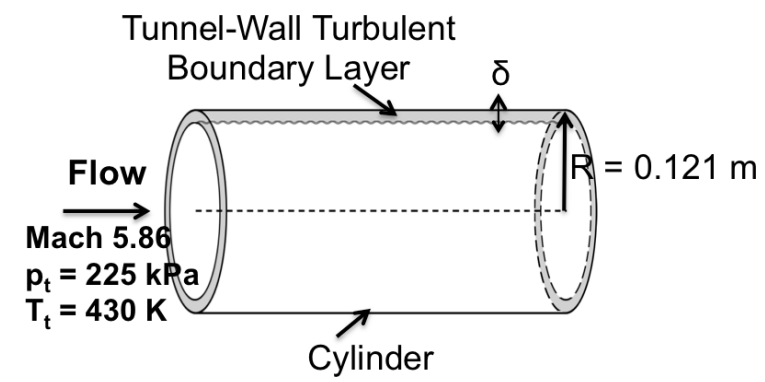

Figure 1. Sketch of computational domain for the current DNS of a turbulent boundary layer inside an axisymmetric cylinder.

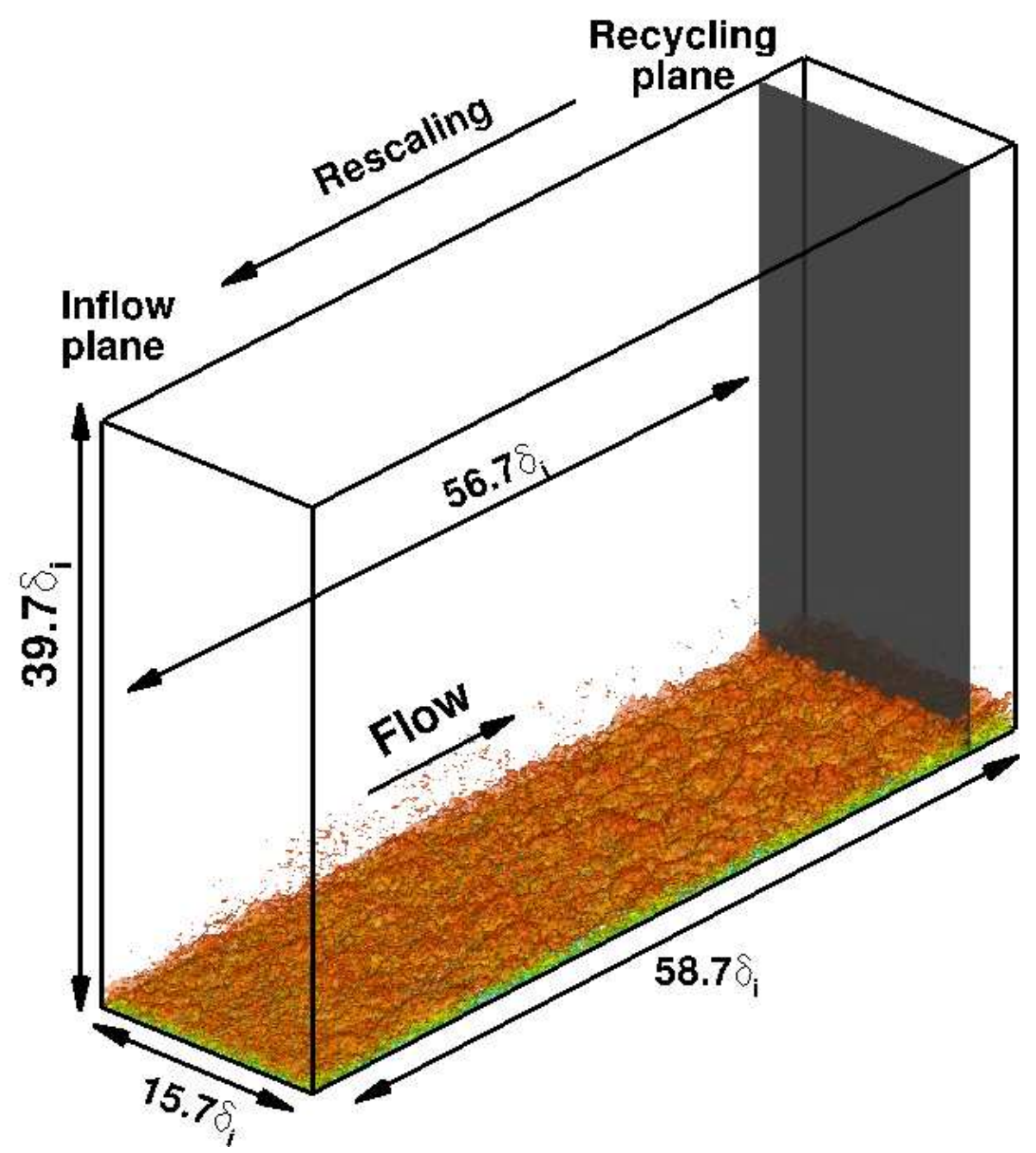

Figure 2. Computational domain and simulation setup of DNS of a Mach 6 turbulent boundary layer on a flat plate. The reference length $\delta_{i}$ is the thickness of the boundary layer (based on $99 \%$ of the freestream velocity) at the inflow plane. An instantaneous flow field is shown in the domain, visualized by an iso-surface of the magnitude of density gradient, $|\nabla \rho| \delta_{i} / \rho_{\infty}=0.98$, colored by the streamwise velocity component (with levels from 0 to $U_{\infty}$, blue to red). 


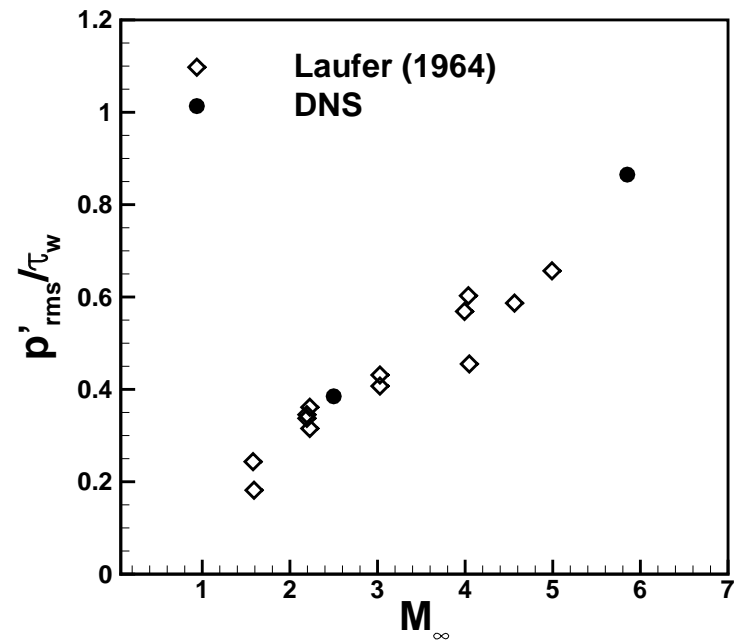

Figure 3. Intensity of freestream pressure fluctuation for DNS compared with the experiments by Laufer. ${ }^{5}$

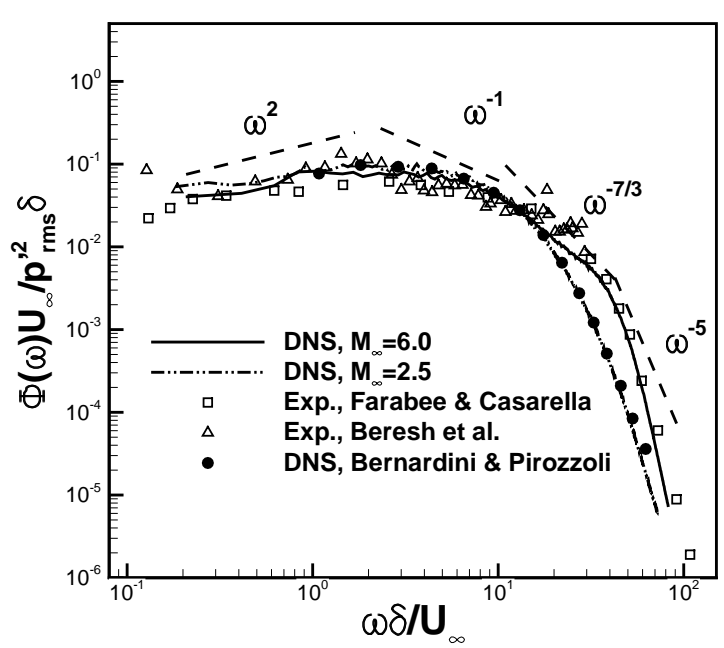

(a) Wall

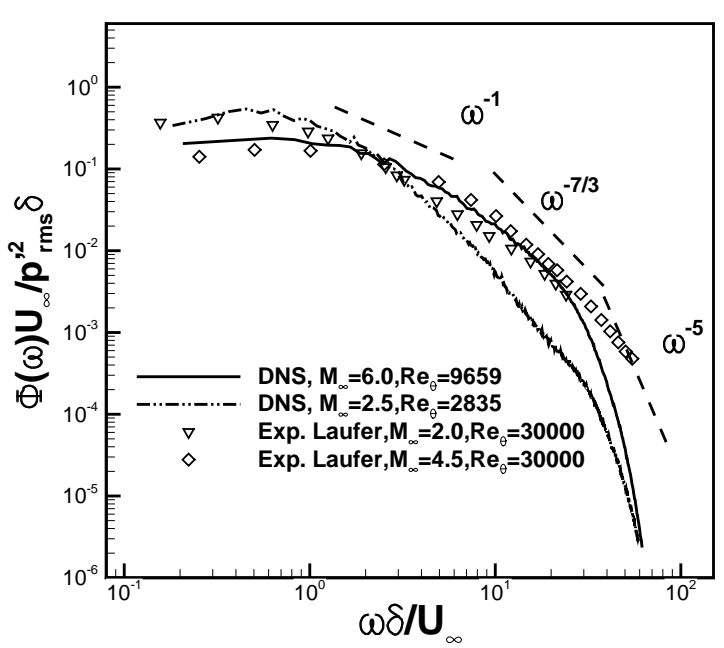

(b) Freestream

Figure 4. Normalized frequency spectrum of computed pressure signal at selected heights. —-: DNS, ${ }^{12,14} \mathrm{M}_{\infty}=2.5$, $\operatorname{Re}_{\tau}=510$; -.-: DNS, $M_{\infty}=5.85, \operatorname{Re}_{\tau}=464$; $\square$ : Farabee \& Casarella, ${ }^{30} M_{\infty} \approx 0, \operatorname{Re}_{\tau}=1169 ; \bullet$ : Bernardini and Pirozzoli, ${ }^{31} M_{\infty}=2, \operatorname{Re}_{\tau}=508 ; \Delta$ : Beresh et al. $,{ }^{9} M_{\infty}=2, \operatorname{Re}_{\tau}=3650 ; \diamond:$ Laufer, ${ }^{5} M_{\infty}=4.5, \operatorname{Re}_{\theta}=30,000$. $\nabla:$ Laufer, ${ }^{5}$ $\mathbf{M}_{\infty}=\mathbf{2 . 0}, \mathbf{R e}_{\theta}=30000$. 


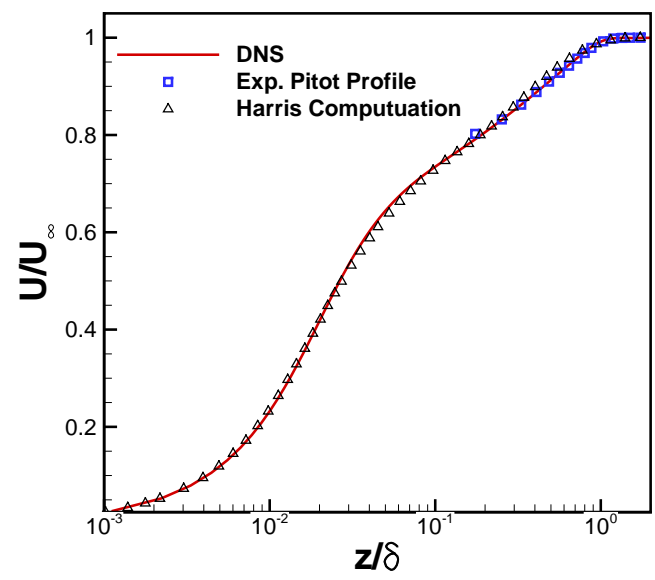

(a)

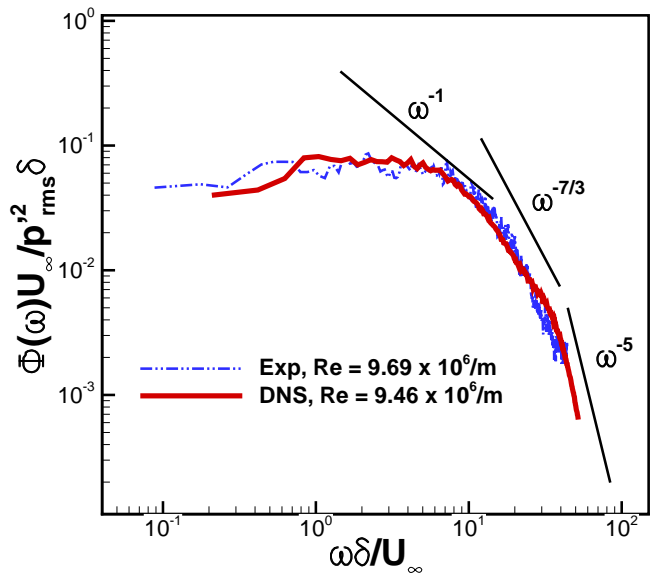

(c)

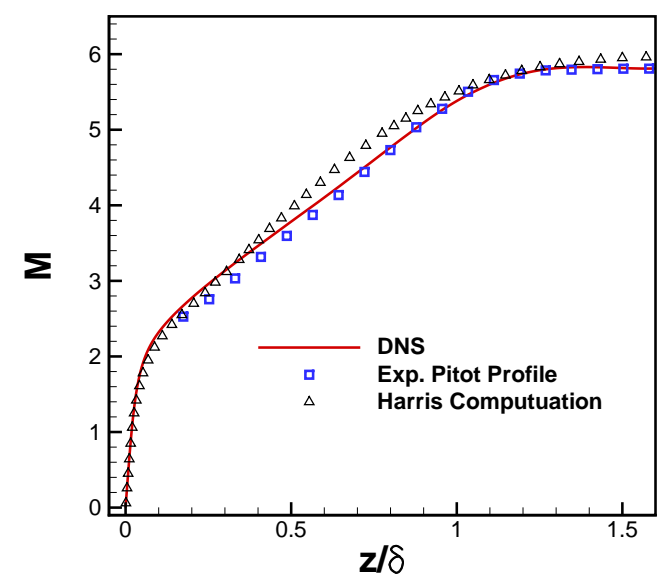

(b)

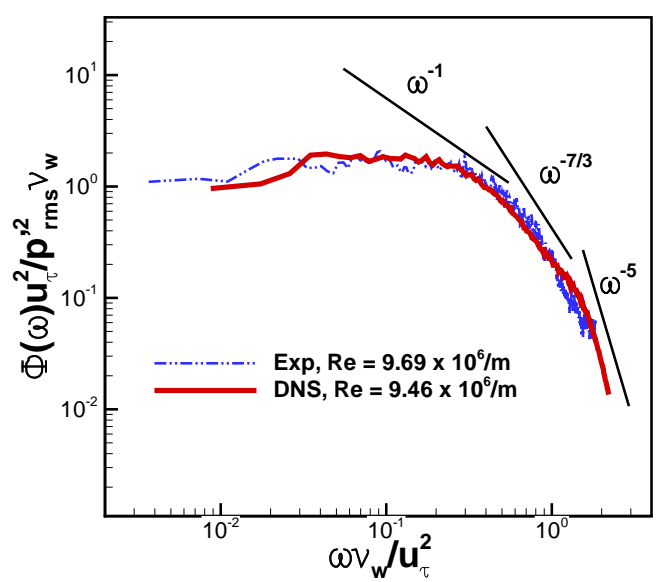

(d)

Figure 5. Comparison of DNS results with those of a Mach-5.8 turbulent boundary layer on the nozzle wall of the Boeing/AFOSR Mach-6 Quiet Tunnel under noisy-flow conditions $\left(R e=9.69 \times 10^{6} / \mathbf{m}, P_{t, \infty}=965 \mathbf{~ k P a}, T_{t, \infty}=429 \mathbf{K}\right)$. The boundary-layer profiles (including experimental Pitot profile and the calculation using Harris boundary-layer $\operatorname{Code}^{34}$ ) were conducted by Steen $^{36}$ and the wall pressure spectrum was measured by Casper. ${ }^{37}$ (a) Mean velocity profile; (b) Mach number profile; (c) frequency spectrum in outer scale; $(d)$ frequency spectrum in inner scale. 


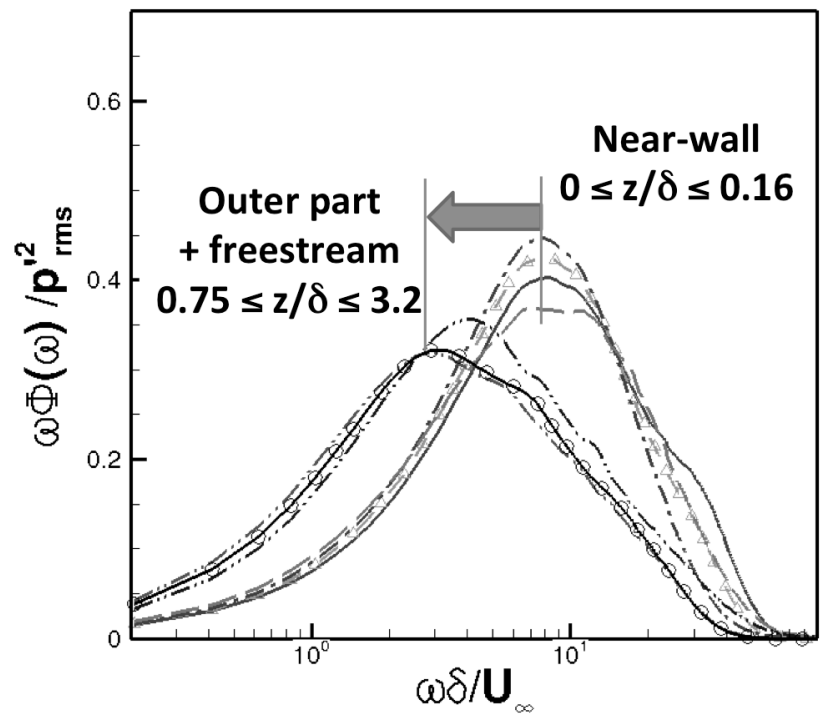

Figure 6. Pre-multiplied power spectrum of pressure signals at at selected heights for the Mach 6 DNS. The pressure spectrum is normalized so that the area under each curve is equal to unity. $z$ is the wall-normal coordinate.

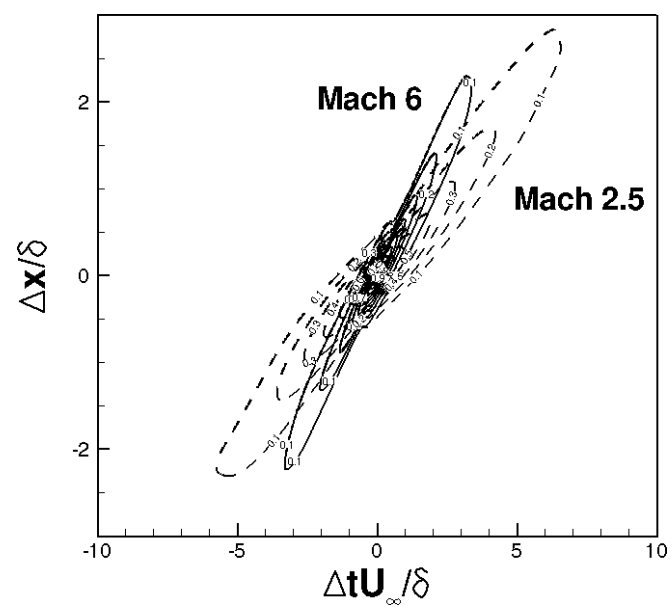

(a)

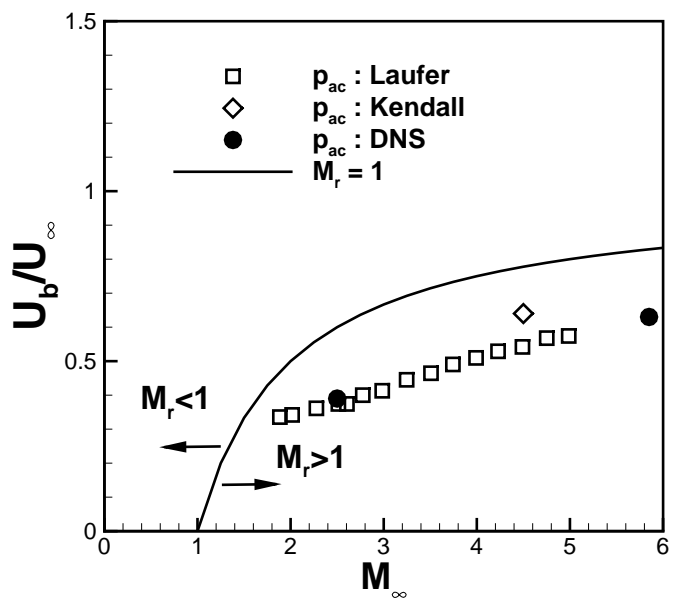

(b)

Figure 7. Mach-number dependence of freestream pressure signal: (a) Space-time correlation coefficient for the Mach 2.5 DNS (Dashed line) and Mach 6 DNS (Solid line). Contour levels vary from 0.1 to 0.9 with increments of 0.1 . (b) Bulk convection speeds of the freestream pressure fluctuation as a function of freestream Mach number. Symbols: $\square$ : Laufer; $^{5} \diamond$ :, Kendall; ${ }^{8} \bullet$ : the present DNS; Lines: $M_{r}=1$. 


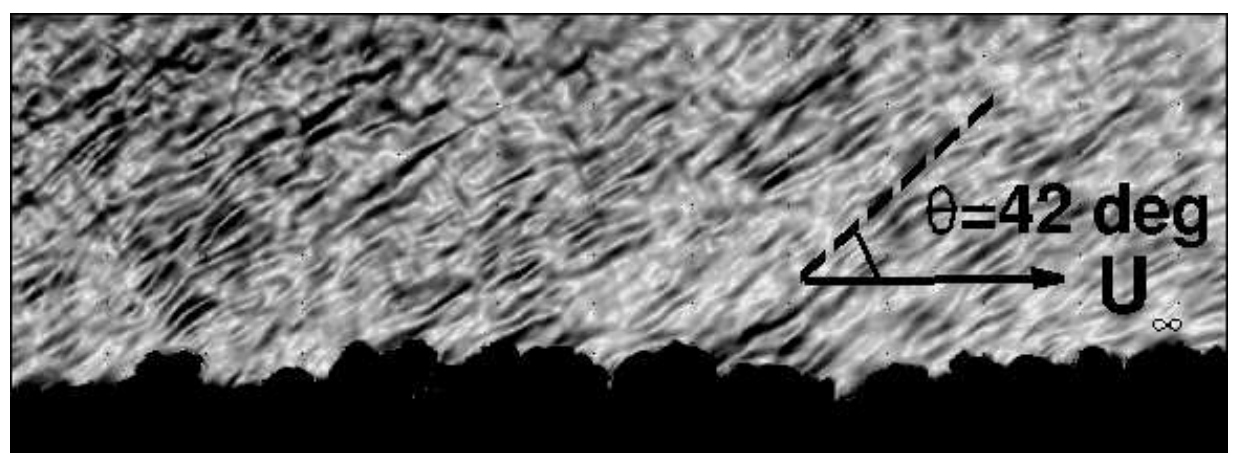

(a)

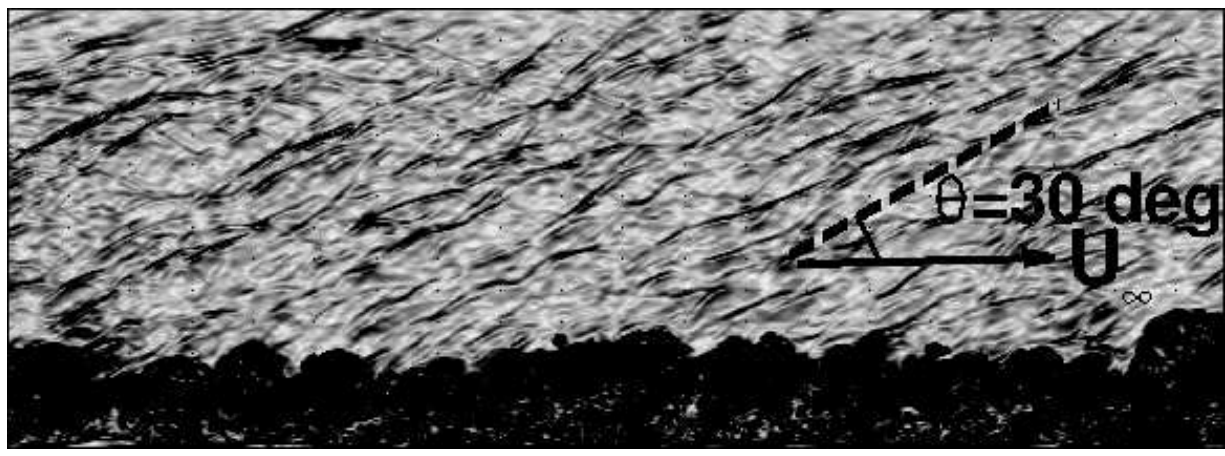

(b)

Figure 8. Numerical schlieren image based on instantaneous flow field: (a) $M_{\infty}=2.5,{ }^{12,14}$ (b) $M_{\infty}=5.85$. Contour levels are selected to emphasize disturbances in the free stream. The dashed line indicates the orientation of the radiation wave front; vector $U$ denotes the flow direction; $\theta$ is the angle between the wave front and $U$. 